\title{
BMJ Open Longitudinal trajectories of suicidal ideation and attempts in adolescents with psychiatric disorders in Chile: study protocol
}

\author{
Pablo Méndez-Bustos (D) ,1,2 Jaime Fuster-Villaseca, ${ }^{3}$ Jorge Lopez-Castroman, ${ }^{4,5,6}$ \\ Oscar Jiménez-Solomon, ${ }^{7,8}$ Cecilia Olivari, ${ }^{1,2}$ \\ Enrique Baca-Garcia (10) 1,9,10,11,12,13,14,15
}

To cite: Méndez-Bustos $P$,

Fuster-Villaseca J, LopezCastroman J, et al. Longitudinal trajectories of suicidal ideation and attempts in adolescents with psychiatric disorders in Chile: study protocol. BMJ Open 2022;12:e051749. doi:10.1136/ bmjopen-2021-051749

- Prepublication history for this paper is available online. To view these files, please visit the journal online (http://dx.doi org/10.1136/bmjopen-2021 051749).

Received 28 March 2021 Accepted 13 January 2022
Check for updates

(C) Author(s) (or their employer(s)) 2022. Re-use permitted under CC BY-NC. No commercial re-use. See rights and permissions. Published by BMJ.

For numbered affiliations see end of article.

Correspondence to

Professor Pablo Méndez-Bustos; p.mendezb@gmail.com

\section{ABSTRACT}

Introduction Adolescent suicide is a worldwide public health problem, being the second and the third leading cause of death in the 15-29 and the 15-19 age groups, respectively. Among adolescents, it is estimated that for every suicide, there are 100-200 suicide attempts. Although $79 \%$ of suicides in the world occur in low/ middle-income countries, most of scientific evidence comes from high-income and low-risk countries. In recent years, adolescent suicide rates have steadily increased in Chile. Deaths caused by self-harm increased by $220 \%$ in the population aged 10-19 years between 2000 and 2015. The Maule Region is one of the regions of Chile with the highest levels of suicide among those aged 15 and 19 years old. The objective of this study is to evaluate the trajectories of ideation and suicidal attempts in adolescents with psychiatric disorders treated within the public health system of the Maule Region, Chile, based on different clinical, psychological and neuropsychological factors.

Method A prospective naturalistic study of a clinical sample of adolescents under psychiatric treatment in the Maule Region, Chile. Adolescents will be evaluated using a thorough protocol that includes suicide-related clinical variables. The study seeks to establish patterns of change in the trajectories of ideation and suicide attempts among adolescents.

Ethics and dissemination Ethical approval was granted by the Scientific Ethics Committee of the Universidad Católica del Maule in Chile. This protocol was registered in ClinicalTrials.gov. The results of this study will be disseminated to health centres through executive reports and feedback sessions. In addition, the most relevant findings will be presented in scientific articles, conferences and seminars open to the community.

Trial registration number NCT04635163.

\section{INTRODUCTION}

Adolescence is the stage of life that extends between childhood and adulthood. Adolescents experience biological growth and development, but also transitions into social roles that have evolved during the last decades. ${ }^{1}$
Strengths and limitations of this study

- The study of longitudinal trajectories of suicidal behaviour has proven to contribute significantly to understanding risk and protective factors of suicidal behaviour.

- Unlike most Chilean studies on adolescent suicide, this study builds on longitudinal data to examine suicidal behaviour trajectories.

- The clinical sample of this study will limit the generalisability of its findings.

Indeed, the period of adolescence has been extended by earlier puberty, longer training, and late marriage and parenthood. ${ }^{2}$ In the present study, following the definition by the WHO and the United Nations, we understand adolescence as the period of life between 10 and 19 years of age. ${ }^{13}$

Few health problems occur in adolescence, but certain behavioural patterns can protect or endanger health in the short or long term. ${ }^{4}$ At this stage, adolescents are in constant search of identity, independence and acceptance. In addition, they are faced with risky situations, which, if left unaddressed, can become factors that promote suicidal behaviour. ${ }^{56}$

Adolescent suicide is a major problem in public health worldwide, and especially concerning in low/middle-income countries. In the Americas, the suicide rate for the age group 10-19years is 3.7 deaths per 100000 population (5.06 for men and 2.31 for women); while in South America, the suicide rate is 3.3 deaths per 100000 people (4.2 for men and 2.4 for women). ${ }^{7}$ In Chile, the suicide rate in this age group for the period 2000-2017 was estimated at 5.4 deaths per 100000 population (for 7.6 men and for 
3.1 women), which is markedly higher than the adolescent suicide rates for the region. ${ }^{8}$

Globally, suicide is the second leading cause of death among young adults aged 15-29 years, after traffic accidents. ${ }^{9}$ For adolescents aged 15-19 years, suicide is the third cause of death. ${ }^{10}$ In the USA, suicide is the second cause of death among adolescents $15-19$ years. ${ }^{11}$ Among Chilean adolescents, $60 \%$ of deaths have intentional causes (suicide and homicide). Deaths by suicide among Chilean adolescents have shown a sustained increase over the last 15 years (55\% between 1999 and 2005), being the second country within the Organization for Economic Co-operation and Development (OECD) with the highest percentage increase. ${ }^{12}$ In a recent study comparing suicide rates among adolescents aged 10-19 years in OECD member countries, Chile had the fourth highest rate with 5.36 deaths per 100000 population. When divided into subgroups, Chile had the highest rates for ages 10-14 years in both men and women with 2.23 and 1.56 deaths per 100000 population, respectively; and in the group of 15-19 years, the third place in men and the fourth in women, with 15.87 and 6.32 deaths per 100000 population. ${ }^{13}$

Adolescence is a critical period of vulnerability for the development of suicidal behaviour, ${ }^{14}$ and the multidimensionality of factors involved-such as biological, genetic, psychiatric, economic, sociodemographic, family related and cultural-makes its study complex. ${ }^{15} 16$ Several studies have shown that adolescents have biological limitations to balance emotions and rationality during decisionmaking. This increased vulnerability seems to be due to an asynchrony in brain development, in which the subcortical limbic system presents a more accelerated development than the orbitofrontal zone. ${ }^{17-19}$ This asynchrony means that when an emotional stimulus is presented, adolescents manifest a higher level of activation in brain areas associated with socioemotional aspects and lower activation in areas related to cognitive control. Rational decision-making is disadvantaged while risk behaviours might be increased by sensation seeking and emotional dysregulation. ${ }^{19-22}$

During this stage, suicidal behaviour shows unstable and fluctuating patterns. ${ }^{23}{ }^{24}$ Suicide rates vary according to gender and age, ${ }^{25}{ }^{26}$ being higher among men than women, while ideation and suicide attempts are more frequent among women. ${ }^{27}$ It is estimated that for each completed suicide in adolescents, there are 100-200 suicide attempts. ${ }^{152829}$ Also, suicidal thoughts have been found to predict later attempts and their repetition. ${ }^{30}$ Approximately one-third of adolescents with suicidal thoughts will make an attempt during the following year and additionally, the lifetime rate of suicidal ideation is $12.1 \%$ in adolescents aged $13-18$ years. ${ }^{24}$ On the other hand, $7.4 \%$ of those aged 10-24years old have reported at least one suicide attempt within the last 12 months with a greater prevalence of attempts among females $(9.3 \%)$ than males $(5.1 \%) .{ }^{24}{ }^{31}$ In Chile, $23.7 \%$ of adolescents aged 13-18 years reported suicidal ideation and $9 \%$ made an attempt in the last 12 months. ${ }^{32}$
The Chilean Health Ministry (MINSAL in Spanish) projected a suicide rate among adolescents aged 10-19 years of 12 deaths per 100000 population for the year $2020{ }^{33}$ The public health objectives for the 2011-2020 decade aimed to diminish this rate by $15 \% .{ }^{34}$ However, in Chile, there are still several limitations and challenges regarding the study of suicidal behaviour in the adolescent population, which makes it difficult to obtain the necessary evidence to plan effective interventions. To obtain useful information, it is necessary to go beyond purely descriptive studies with cross-sectional designs and implement studies that formulate explanatory models, along with evaluating the evolution of suicidal behaviour in adolescents. ${ }^{35}$ Considering the risk of onset of suicidal thoughts and attempts during adolescence and the variability in their evolution, it becomes necessary to make an early and timely identification of high-risk clinical subgroups. The analysis of suicidal patterns over time and the effect of specific risk factors could meet such objective.

\section{Aims of the project}

The primary aim of this study will be to assess ideation trajectories and suicide attempts among adolescent outpatients with psychiatric disorders followed by the public health system of the Maule Region, Chile. Our secondary aims will be to: (1) identify clinical, psychological, and neuropsychological factors for suicidal ideation and attempts during the follow-up period; (2) estimate the predictive value of clinical, psychological, and neuropsychological factors on the course of suicidal ideation and attempts in adolescent psychiatric outpatients; (3) identify and characterise groups (latent classes) of adolescent patients with psychiatric disorders based on their longitudinal patterns of suicidal ideation and suicide attempts; and (4) identify and characterise groups of adolescent patients with psychiatric disorders, according to repetition of suicide attempts.

\section{METHODS AND ANALYSIS}

\section{Study design}

Prospective naturalistic study in a clinical sample.

\section{Characteristics of the study context}

The Maule Region is 1 of the 16 regions that compose the Republic of Chile; it is located in the central zone of the country, extending over a territory of $30269 \mathrm{~km}^{2}$ and it is also the fourth most populated region with 1044950 inhabitants. ${ }^{37}$ Administratively, the region comprises four provinces (Curicó, Talca, Linares and Cauquenes) which are, in turn, subdivided into 30 communes, with Talca being the region's capital city. ${ }^{38}$

The Maule Health Service (SSMaule, from the Spanish Servicio de Salud Maule) is the public health network for the region. It is composed of 13 hospitals, 30 health departments, 158 rural emergency centres, 5 community centres for mental health (COSAM, Centros Comunitarios 
de Salud Mental) and 41 family health centres (CESFAM, Centros de Salud Familiar) which are part of the Primary Healthcare Network of the Maule Region (APS, Sistema de Atención Primaria de Salud). ${ }^{39}$ The SSMaule manages a Mental Health Network integrated by multidisciplinary teams within the Public Health Network. The APS provides mental health prevention and treatment programmes available to all the people in the region. Patients with the most severe mental health problems are referred, via consultation, from the APS to the mental health and psychiatric units in hospitals. ${ }^{40}$

During the period 2014-2019, there was a significant increase, with respect to the prior 5 years, in the number of mental health visits at a regional and national level: adolescent visits increased by $34 \%$ at a national level and $48 \%$ at a regional level. The communes of Talca, Curicó and Linares reported a $56.7 \%$ increase in this age group. ${ }^{41}$

\section{Sample design}

Adolescents shall be selected by means of non-probability purposive sampling.

Inclusion criteria:

- Adolescents between 10 and 19 years of age.

- With a diagnosis of psychiatric disorder (with or without suicidal ideation or attempts).

- Who are being treated in mental health units of hospitals, CESFAM or COSAM in the Maule Region.

Exclusion criteria:

- Adolescents unable to read or write.

- Adolescents with a cognitive disability.

- Adolescents with symptoms of active psychosis.

\section{Instruments}

The following instruments compose the study protocol. They were selected because they have been used in prior studies related to adolescent suicidal behaviour. Spanishlanguage versions of these instruments will be used.

\section{Barrat's Impulsiveness Scale}

The Barrat's Impulsiveness Scale is a scale for assessing impulsiveness, composed of 30 items grouped into three subscales: cognitive impulsiveness ( 8 items), motor impulsiveness (10 items) and non-planned impulsiveness (12 items). Each item uses a 4-point Likert response format (1=rarely or never; 2=occasionally; $3=$ often; $4=$ always or nearly always). This scale has been validated in the Chilean adolescent population with acceptable internal consistency (Cronbach's alpha $=0.77){ }^{42}$

\section{Depression, Anxiety and Stress abbreviated Scale}

The Depression, Anxiety and Stress abbreviated Scale (DASS-21) is a scale composed of 21 items which measure the presence of depressive symptomatology (7 items), anxious symptomatology ( 7 items) and stress (7 items). Each statement has four alternative answers in Likert format describing how the assessed person felt during the last week ( $0=$ nothing; $1=\mathrm{a}$ little; $2=$ quite; $3=\mathrm{alot}$ ). The DASS-21 has been validated among the Chilean population with high internal consistency (Cronbach's alpha $=0.91) .{ }^{43}$

\section{Insomnia Severity Index}

The Insomnia Severity Index scale was designed as a screening tool to determine the clinical significance of insomnia, by the assessment of sleep difficulties in the last 2 weeks. This instrument consists of seven items with responses in a 5-point Likert format. The resulting score is classified into insomnia without clinical importance, mild insomnia, moderate insomnia and severe insomnia. This instrument has shown adequate internal consistency (Cronbach's alpha $=0.82) .{ }^{44}$

\section{Difficulties in Emotion Regulation Scale}

The Difficulties in Emotion Regulation Scale is an instrument which measures clinically relevant difficulties in emotional regulation, and it includes five subscales: emotional control difficulties (six items), emotional rejection (seven items), emotional interference (four items), emotional inattention (five items) and emotional confusion (three items). Response categories follow a 6-point Likert format (1=totally disagree; 2=quite disagree; $3=$ somehow disagree; $4=$ somehow agree; $5=$ quite agree; $6=$ totally agree). This scale has been validated in Chile among university population with high internal consistency (Cronbach's alpha=0.92). ${ }^{45}$

\section{Brief Parental Scale}

The Brief Parental Scale has three subscales with a 5-point Likert-type answer format (1=highly disagree, $2=$ disagree, $3=$ notsure, $4=$ agree, $5=$ highly agree). It assesses four dimensions: responsiveness, warmth, demand and parental monitoring. This scale has been validated among the Chilean adolescent population with high internal consistency (Cronbach's alpha between 0.81 and 0.87 for each dimension). ${ }^{46}$

\section{General Help Seeking Questionnaire for mental health problems in adolescents}

This questionnaire has 11 items describing perceived barriers for seeking professional help. Each item is assessed by a 6 -point Likert scale ( $1=$ strongly disagree to $6=$ strongly agree). This scale has been validated in Chile among the adolescent population; it showed moderatehigh internal consistency (Cronbach's alpha $=0.76){ }^{47}$

\section{lowa Gambling Task}

The aim of this test is to assess the process of decisionmaking. The person must choose among four groups of cards labelled A, B, C, D. The person being assessed starts the 'game' with a simulated amount of money and the objective is to get a maximum increase of such quantity and try to lose as little as possible. It has been used with adolescents and showed a high sensitivity when measuring deficiencies in decision-making and their relation with suicidal behaviour. ${ }^{48}$ 


\section{University of Columbia Suicide Severity Rating Scale}

This instrument was designed with the goal of gathering information about suicidal ideation and behaviour, as well as non-suicidal self-injuries. It allows quantifying the severity of both suicidal ideation intensity and behaviour lethality along specific time periods. This instrument has a mixed format, with open, closed and Likert-format questions. The University of Columbia Suicide Severity Rating Scale has acceptable predictive power for future suicidal attempts for clinical and adolescent populations. ${ }^{49} 50$

\section{Procedure and application}

Information about the study will be disseminated among mental health units of hospitals, COSAM and CESFAM in the Maule Region. A registry of patients attending mental health units during the first 6 months of the study will be created to facilitate recruitment and follow-up. The treating clinical team will provide information about the study to their patients; and if they agree to participate, the researchers will contact them, always protecting patient anonymity. Participants will receive written and verbal information about the essential aspects of the research, its objective, benefits and clinical implications. Informed consent, signed by the participant if older than 18 years of age and by the mother, father, or responsible tutor if under 18, will be requested subsequently. Selected patients will be assessed at three time points during the study: at baseline, at 6 months and 6 months after the second measurement. It is estimated that the implementation time of the protocol will range from 60 to $80 \mathrm{~min}$.
To complement the information collected with the study protocol, clinical records will be used to extract data on family history, mental illnesses, hospitalisations, treatments and previous suicide attempts. In this study, suicide attempts will be understood as an act in which a person hurts themselves, with the intention of dying, and survives. ${ }^{51}$

Please review figure 1 describing the phases involved in the implementation of the study protocol.

\section{Statistical analysis and results}

Initially, different exploratory analyses will be carried out with the goal of obtaining a first approximation to the data and spot possible coding errors. Then, we will conduct analyses to examine the reliability and validity for each of the instruments in our protocol. Modelling techniques will also be implemented with the objective of analysing the information gathered from an explicative and predictive perspective, exploring the extent to which the models being tested fit the data.

Finally, using latent class analyses for longitudinal data, we will seek to identify change trajectories in relation to the variables studied, based on the following hypotheses:

1. High levels of suicidal ideation at baseline will be associated with a higher likelihood of suicide attempts during follow-up.

2. Adolescents with suicidal ideation and several previous attempts will be more likely to reattempt than those with a single attempt.

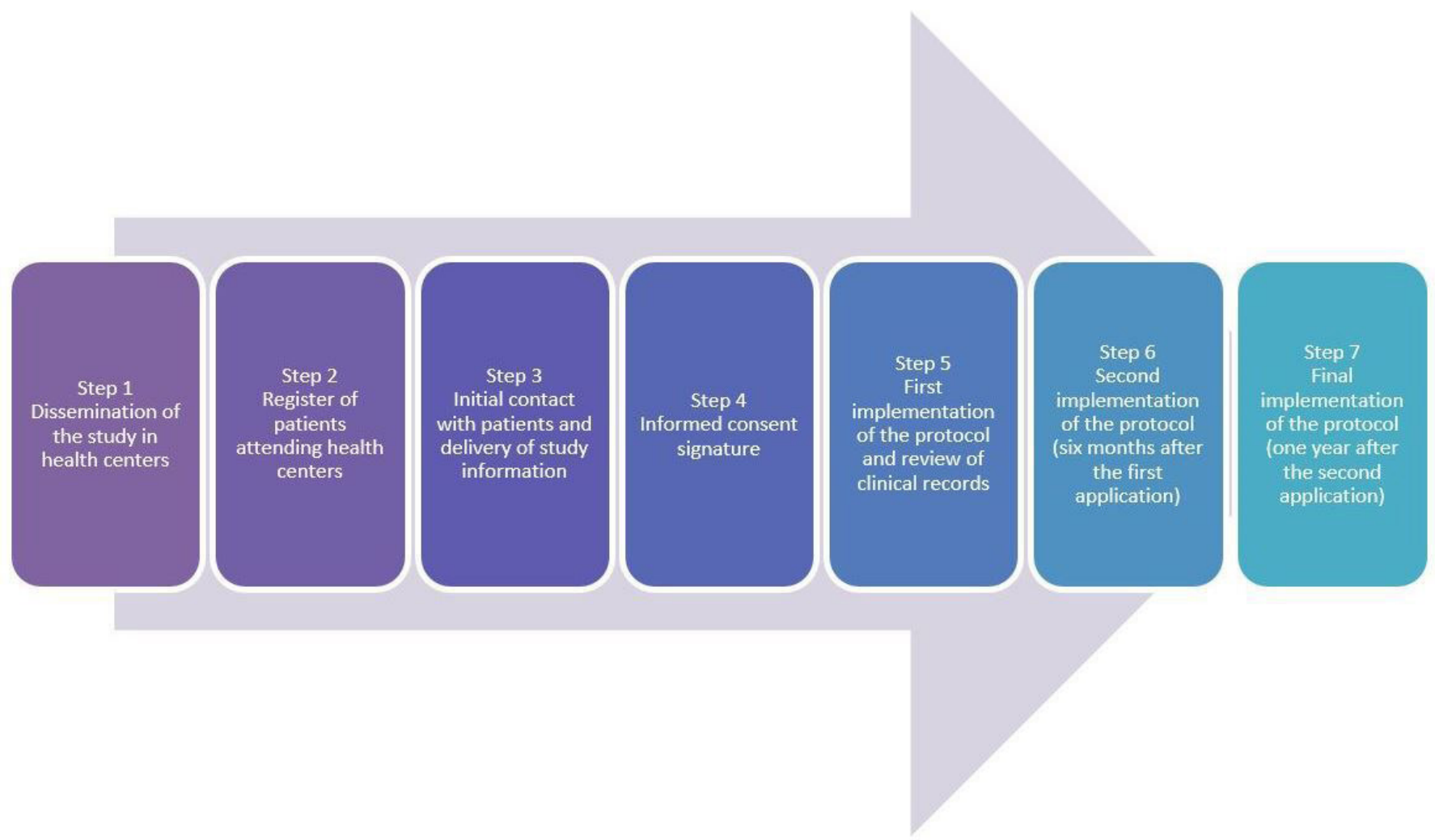

Figure 1 The seven steps of the procedure and implementation of the study protocol. 
3. Adolescents with less emotional regulation, poor parental styles, high levels of impulsivity and a history of self-harm will have an irregular course of suicidal thoughts and will be less likely to ask for help.

4. Adolescents with a history of suicide attempts, psychiatric comorbidity and impaired decision-making will show higher levels of suicidal ideation and will be more likely to reattempt than those without these characteristics.

\section{Participation}

To achieve reliable estimators in models examining trajectories of change over time, it is recommended to use a minimum number of 100 cases. $^{52}$ In this study, we aim to recruit a minimum sample of 300 adolescents to fulfil the objectives and proposed statistical analyses. With this goal and to minimise attrition, the principal investigator will arrange clinical meetings with participants' teams or providers (physicians or psychologists) during the research and the follow-up period (24 months). Patients will also be contacted by phone 48 hours before each evaluation, to confirm their participation. In addition, participants' transportation costs will be paid by the study, since many of them live far from the health centres.

\section{Patient and public involvement}

Clinical personnel at the health centres (physicians or psychologists) will deliver study information to patients and will obtain informed consent from participants (or their parents or legal guardians for those under 18 years of age) and implement all research protocols. No patients were involved in the design, preparation or implementation of this study.

\section{ETHICS, DATA MANAGEMENT AND DISSEMINATION \\ Ethical review}

The research project was approved by the Ethics Committee of the Maule Health Service and by the Scientific Ethics Committee of the Universidad Católica del Maule de Chile, meeting principles established by the Universal Declaration of Human Rights, international regulations of the Council for International Organizations of Medical Sciences and Law 20120 regarding scientific research involving human beings in Chile. ${ }^{53}$ Also, the authors declare that necessary ethical safeguards have been adopted for working with people, as proposed in the Helsinki Declaration and the Belmont report. ${ }^{55} 56$

To safeguard the anonymity of the participants, no work or publication associated with this study will disclose data that could allow the identification of the participants. Each person who is part of the study will be anonymised with a record number that will allow their identity to be hidden.

No financial compensation will be provided to participants. Any benefits that participants obtain derive from the information they provide, the understanding of behavioural patterns and the identification of clinical subgroups with high suicidal risk.

Since the instruments used in the study are commonly used in clinical evaluation, they do not carry significant risk or limitations for patients. Previous studies have already shown that repeatedly asking patients at risk of suicide does not increase the likelihood that they will engage in suicidal behaviour. ${ }^{5758}$

Prior to evaluation, each participant must indicate their authorisation through written consent. In addition, participants under the age of 18 years must have the written permission of their parents or guardians.

\section{Data management}

The processing of data is carried out under a strict safeguard of the confidentiality and anonymity of the information.

Each evaluator will be responsible for entering the information into a link on a Google Forms platform, which is managed exclusively by the lead researcher. This platform has an encryption security system .

After participants have entered the information collected in the evaluation sessions, the principal investigator will export the data in a Microsoft Excel-compatible document, where he will remove any personal information that could allow the identification of participants, assigning a record number to each case entered. The resulting document will be delivered to the research team for further cleaning and analysis. All cases with more than $5 \%$ missing data will be eliminated. Missing values in the remaining cases will be handled by multiple imputation using the linear regression method, considering restrictions for the possible values of each scale. Multiple imputation will be carried out for each instrument separately.

\section{Dissemination}

Study findings will be disseminated via scientific publications, presentations at conferences, lectures in congresses, activities open to the community and seminars with health teams of the Maule Region, Chile. Also, summary reports will be prepared and distributed among providers of health units and centres that participated in the study, with the goal of providing information which could be incorporated in the mental health assessment and treatment of adolescents at risk of suicide.

\section{DISCUSSION}

Adolescent suicide is the consequence of a complex interaction among genetic, biological, psychiatric, psychological, social and cultural factors. Though there is consensus regarding the importance of developing more effective interventions for reducing suicide risk among adolescents, there are still many limitations and challenges in the detection and early and timely prevention of suicidal thoughts and attempts.

Reviews of the existing evidence have pointed out that models proposed in the last 50 years present a low 
predictive capacity and that risk factor categories have become homogeneous, rendering them inadequate to identify specific groups of factors associated with the different types of suicidal behaviour. ${ }^{36}$

Considering the risk of onset of ideation and suicidal attempts during adolescence and the variability in their evolution, it is of paramount importance to identify vulnerable groups early and timely. Therefore, it is vital to use methodological approaches that help overcome the weaknesses of cross-sectional studies and build specific explanatory models that allow a deeper understanding of the development and trajectory of suicidal ideation and attempts.

The methodology proposed for this study will seek to overcome the limitations of cross-sectional and retrospective studies by identifying patterns of suicidal thoughts and behaviours for a specified period and the effect and interactions of specific clinical, psychological and neuropsychological factors.

While the number of instruments in our protocol is extensive, the questionnaires to be employed consist of short answers which are easy to comprehend. Furthermore, each questionnaire will be applied by a trained research assistant (psychologist) and each question will be read to the adolescent to ensure understanding and quality of the answer.

Although the use of protocols in clinical practice has been questioned due to their low predictive capacity, ${ }^{59}$ such protocols can improve efficiency and consistency in decision-making, allowing the development of an individualised treatment plan and better risk management.

Finally, deaths by self-inflicted injuries are irregularly distributed around the world. While $79 \%$ of the world's suicides occurred in low/middle-income countries, ${ }^{10}$ high-income countries had the highest rate, at 11.5 per 100000 . Nearly three times as many men as women die by suicide in high-income countries, in contrast to low/ middle-income countries, where the rate is more even. ${ }^{60}$ It is paradoxical that around $82 \%$ of scientific production comes from North America and Europe, considering that countries such as China and India account for almost half of suicides worldwide and some Latin American countries exceed the European average. ${ }^{761}$ It is necessary to promote more studies in these regions of the world and to identify cultural and other regional and country-specific factors that can improve the understanding of this public health problem.

Finally, this study also seeks to help overcome the limited ability of current research to identify and compare diverse patterns of suicidal ideation and behaviour in adolescents. Thus, we hope that our study will contribute to improve the understanding of adolescent suicide risk trajectories and to develop interventions focused on subclinical groups with higher risk, while considering important cultural and local factors.

\section{Author affiliations}

${ }^{1}$ Department of Psychology, Universidad Católica del Maule, Talca, Chile
${ }^{2}$ Faculty of Health Sciences, Universidad Católica del Maule, The Neuropsychology and Cognitive Neurosciences Research Center (CINPSI Neurcog), Talca, Chile ${ }^{3}$ Doctoral Programme in Psychology, Universidad Católica del Maule, Talca, Chile ${ }^{4}$ Department of Adult Psychiatry, Nîmes University Hospital, Nimes, France ${ }^{5}$ IGF, CNRS-INSERM, University Montpellier, Montpellier, France

${ }^{6}$ CIBERSAM, Madrid, Spain

${ }^{7}$ New York State Center of Excellence for Cultural Competence, New York State Psychiatric Institute, Columbia University Medical Center, New York, New York, USA ${ }^{8}$ Center on Poverty and Social Policy, School of Social Work, Columbia University, New York, New York, USA

${ }^{9}$ Psychiatry, University Hospital Jimenez Diaz Foundation, Madrid, Spain

${ }^{10}$ Psychiatry, Autonomous University of Madrid, Madrid, Spain

${ }^{11}$ Department of Psychiatry, University Hospital Rey Juan Carlos, Mostoles, Spain

${ }^{12}$ Department of Psychiatry, General Hospital of Villalba, Villalba, Spain

${ }^{13}$ Department of Psychiatry, University Hospital Infanta Elena, Valdemoro, Spain

${ }^{14}$ CIBERSAM (Centro de Investigacion en Salud Mental), Carlos III Institute of Health,

Madrid, Spain

${ }^{15}$ Department of psychiatry, Centre Hospitalier Universitaire de Nîmes, Nîmes, France

Contributors PM-B planned and designed the study and drafted the article. JF-V and JL-C contributed to the design of the study and drafted the article. OJ-S, CO and EB-G supervised the development of this manuscript and provided editorial input. All authors critically revised the article, gave their final approval to the manuscript, and agree to be accountable for all aspects of the work in ensuring that questions related to the accuracy or integrity of any part of the work are appropriately investigated and resolved.

Funding This study was supported by the National Fund for Scientific and Technological Development (Fondo Nacional de Desarrollo Científico y TecnológicoFONDECYT No.11170342) of the Government of Chile.

\section{Competing interests None declared.}

Patient and public involvement Patients and/or the public were not involved in the design, or conduct, or reporting, or dissemination plans of this research.

Patient consent for publication Not required.

Provenance and peer review Not commissioned; externally peer reviewed.

Open access This is an open access article distributed in accordance with the Creative Commons Attribution Non Commercial (CC BY-NC 4.0) license, which permits others to distribute, remix, adapt, build upon this work non-commercially, and license their derivative works on different terms, provided the original work is properly cited, appropriate credit is given, any changes made indicated, and the use is non-commercial. See: http://creativecommons.org/licenses/by-nc/4.0/.

ORCID iDs

Pablo Méndez-Bustos http://orcid.org/0000-0002-9026-6974

Enrique Baca-Garcia http://orcid.org/0000-0002-6963-6555

\section{REFERENCES}

1 WHO. Adolescent health. world heal. organ, 2021. Available: https:// www.who.int/health-topics/adolescent-health\#tab=tab_1 [Accessed 11 Mar 2021].

2 Sawyer SM, Azzopardi PS, Wickremarathne D, et al. The age of adolescence. Lancet Child Adolesc Health 2018;2:223-8.

3 UNFPA. Adolescent and youth demographics: a brief overview. New York, NY, 2013. Available: https://www.unfpa.org/sites/default/files/ resource-pdf/One pager on youth demographics GF.pdf

4 Cuesta-Revé D. Aspectos epidemiológicos del suicidio en adolescentes. Rev Mex Pediatr 2017;84:72-7.

5 Martin-Fumadó C, Gómez-Durán EL. Suicide investigation: psychological autopsy. Spanish J Leg Med 2017;43:135-7.

6 Suelves JM, Robert A. La conducta suicida: una mirada desde La salud pública. Rev Española Med Medicina Legal 2012;38:137-42.

7 PAHO. Suicide mortality in the Americas: regional report. Washington, DC: Pan American Health Organization, 2014. https:// www.paho.org/hq/dmdocuments/2015/suicide-PAHO-mortality2014.pdf

8 Araneda N, Sanhueza P, Pacheco G, et al. Suicidio en adolescentes y jóvenes en Chile: riesgos relativos, tendencias y desigualdades. Rev Panam Salud Pública 2021;45:1-9. 
9 WHO. Preventing suicide: a global imperative. World Health Organization: Ginebra, Suiza, 2014. https://apps.who.int/iris/ bitstream/handle/10665/131056/9789241564779_eng.pdf? sequence $=1$

10 WHO. Suicide, 2019. Available: https://www.who.int/news-room/ fact-sheets/detail/suicide [Accessed 2 Sep 2019].

11 Shain B, Braverman PK, Adelman WP, Committee on adolescence. Suicide and suicide attempts in adolescents. Pediatrics 2016;138:e20161420.

12 OECD. Health at a glance. Paris: OECD, 2011

13 Roh B-R, Jung EH, Hong HJ. A comparative study of suicide rates among 10-19-Year-Olds in 29 OECD countries. Psychiatry Investig 2018:15:376-83.

14 Breton J-J, Tousignant M, Bergeron L, et al. Informant-specific correlates of suicidal behavior in a community survey of 12 - to 14-year-olds. J Am Acad Child Adolesc Psychiatry 2002;41:723-30.

15 Kennebeck S, Bonin L. Suicidal behavior in children and adolescents: Epidemiology and risk factors. UptoDate [online database], 2017. Available: https://www.uptodate.com/contents/suicidal-behavior-inchildren-and-adolescents-epidemiology-and-risk-factors?topicRef= 4867

16 Wasserman D, Rihmer Z, Rujescu D, et al. The European psychiatric association (EPA) guidance on suicide treatment and prevention. Eur Psychiatry 2012;27:129-41.

17 Padrón I, Rodrigo MJ, De-Vega M. Búsqueda de predictores del proceso de toma de decisiones de riesgo en situaciones sociales en La adolescencia. Rev Estud e Investig en Psicol y Educ 2015;2.

18 Rodrigo MJ, Padrón I, de Vega M, et al. Neural substrates of counterfactual emotions after risky decisions in late adolescents and young adults. $J$ Res Adolesc 2018;28:70-86.

19 Casey B, Jones RM, Somerville LH. Braking and accelerating of the adolescent brain. J Res Adolesc 2011;21:21-33.

20 Broche Y. Modelo de desbalance del desarrollo cerebral: nuevo enfoque teórico en La comprensión de conductas de riesgo en La adolescencia. Rev Cuba Neurol y Neurocir 2015;5:s38-44 http:// www.revneuro.sld.cu/index.php/neu/article/view/184

21 Lee WK, Lim D, Lee HA, et al. Sensation seeking as a potentia screening tool for suicidality in adolescence. BMC Public Health 2015;16:92

22 Hatkevich C, Penner F, Sharp C. Difficulties in emotion regulation and suicide ideation and attempt in adolescent inpatients. Psychiatry Res 2019;271:230-8.

23 Miranda R, Shaffer D. Understanding the suicidal moment in adolescence. Ann N Y Acad Sci 2013;1304:14-21.

24 Nock MK, Green JG, Hwang I, et al. Prevalence, correlates, and treatment of lifetime suicidal behavior among adolescents. JAMA Psychiatry 2013;70:300.

25 McKay K, Milner A, Maple M. Women and suicide: beyond the gender paradox. Int J Cult Ment Health 2014;7:168-78.

26 Schrijvers DL, Bollen J, Sabbe BGC. The gender paradox in suicidal behavior and its impact on the suicidal process. J Affect Disord 2012;138:19-26.

27 McLoughlin AB, Gould MS, Malone KM. Global trends in teenage suicide: 2003-2014. QJM 2015;108:765-80.

28 Sarchiapone M, D'Aulerio M, losue M. Suicide attempts and completed suicide in adolescents: neurobiological aspects. Adv Biol Psychiatry 2016;30:11-20.

29 Wasserman D, Carli V, Durkee T, et al. S39-01 - Epidemiology of suicidal and risk-taking behavior. European Psychiatry 2010;25:83

30 Miranda R, De Jaegere E, Restifo K, et al. Longitudinal follow-up study of adolescents who report a suicide attempt: aspects of suicidal behavior that increase risk of a future attempt. Depress Anxiety 2014;31:19-26.

31 Annor FB, Clayton HB, Gilbert LK, et al. Sexual orientation discordance and nonfatal suicidal behaviors in U.S. high schoo students. Am J Prev Med 2018:54:530-8.

32 Silva $D$, Vicente $B$, Arévalo $E$, et al. Intento de suicidio $Y$ factores de riesgo en Una muestra de adolescentes escolarizados de Chile. RPPC 2017;22:33-42.

33 MINSAL. Programa nacional de prevención del suicidio: orientaciones para su implementación. Santiago: Ministerio de Salud, 2013.

34 MINSAL. Estrategia Nacional de Salud para el cumplimiento de los objetivos sanitarios de la década 2011-2020. Santiago, Ministerio de Salud, 2011. Available: https://www.minsal.cl/portal/url/item/c403 4eddbc96ca6de0400101640159b8.pdf

35 Belsher BE, Smolenski DJ, Pruitt LD, et al. Prediction models for suicide attempts and deaths. JAMA Psychiatry 2019;76:642-11.
36 Franklin JC, Ribeiro JD, Fox KR, et al. Risk factors for suicidal thoughts and behaviors: a meta-analysis of 50 years of research. Psychol Bull 2017;143:187-232.

37 INE. Resultados definitivos Censo 2017. Santiago de Chile: Instituto Nacional de Estadísticas, 2017. Available: http://www.censo2017.cl/ wp-content/uploads/2017/12/Presentacion_Resultados_Definitivos_ Censo2017.pdf

38 Región del Maule, 2018. Available: https://www.gob.cl/regiones/ region-del-maule/

39 SSMaule. ¿Quienes somos? Talca, Chile: Servicio de Salud Maule, 2018. Available: https://www.ssmaule.cl/minsal/?page_id $=122$

40 SSMaule. Salud mental. Talca, Chile: Servicio de Salud Maule, 2012. Available: https://www.ssmaule.cl/minsal/?p=375

41 Departamento de Estadísticas e Información de Salud [DEIS]. Reportería Programa Salud Mental. Año 2014-2020, 2020. Available: http://www.deis.cl/wp-content/2018/gobCL-sitios-1.0/assets/ Dashboard SaludMental.html

42 Salvo G L, Castro S A. Confiabilidad Y validez de la escala de impulsividad de Barratt (BIS-11) en adolescentes. Rev Chil Neuropsiquiatr 2013;51:245-54.

43 Antúnez Z, Vinet E, Depresión Ede. (DASS - 21): Validación de la Versión abreviada en Estudiantes Universitarios Chilenos. Ter psicológica 2012:30:49-55.

44 Fernandez-Mendoza J, Rodriguez-Muñoz A, Vela-Bueno A, et al. The Spanish version of the insomnia severity index: a confirmatory factor analysis. Sleep Med 2012:13:207-10.

45 Guzmán-González M, Trabucco C, Urzúa M A, et al. Validez Y Confiabilidad de la Versión Adaptada al Español de la Escala de Dificultades de Regulación Emocional (DERS-E) en Población Chilena. Ter Psicol 2014;32:19-29.

46 Cumsille P, Martínez ML, Rodríguez V, et al. Análisis Psicométrico de la Escala parental breve (EPB): Invarianza Demográfica Y longitudina en Adolescentes Chilenos. Psykhe 2014;23:1-14.

47 Olivari C, Guzmán-González M. Validación del cuestionario General de búsqueda de ayuda para problemas de salud mental en adolescentes. Rev chil. pediatr. 2017;88:324-31.

48 Bridge JA, McBee-Strayer SM, Cannon EA, et al. Impaired decision making in adolescent suicide attempters. J Am Acad Child Adolesc Psychiatry 2012;51:394-403.

49 Gipson PY, Agarwala P, Opperman KJ. Columbia-Suicide severity rating scale: predictive validity with adolescent psychiatric emergency patients. Pediatr Emerg Care 2014;31:88-94.

50 Posner K, Brown GK, Stanley B, et al. The Columbia-Suicide severity rating scale: initial validity and internal consistency findings from three multisite studies with adolescents and adults. Am J Psychiatry 2011;168:1266-77

51 De Leo D, Goodfellow B, Silverman M, et al. International study of definitions of English-language terms for suicidal behaviours: a survey exploring preferred terminology. BMJ Open 2021;11:1-10.

52 Curran PJ, Obeidat K, Losardo D. Twelve frequently asked questions about growth curve modeling. J Cogn Dev 2010;11:121-36.

53 Kottow M. Regulación en bioética y la necesaria participación. Rev Chil Salud Pública 2012;16:10.

54 van Delden JJM, van der Graaf R. Revised CIOMS international ethical guidelines for health-related research involving humans. JAMA 2017:317:135

55 World Medical Association (WMA). WMA Declaration of Helsink - Ethical Principles for Medical Research Involving Human Subjects, 2018. Available: https://www.wma.net/policies-post/ wma-declaration-of-helsinki-ethical-principles-for-medical-researchinvolving-human-subjects/ [Accessed 22 May 2020].

56 Zucker D. The Belmont report. en: Wiley encyclopedia of clinical trials. Hoboken, NJ, USA: John Wiley \& Sons, Inc, 2007.

57 WHO. Preventing suicide: a resource for counsellors. Geneva: World Health Organization, 2006. https://apps.who.int/iris/bitstream/handle/ 10665/43487/9241594314_eng.pdf?sequence=1\&isAllowed=y

58 Husky M, Olié E, Guillaume S, et al. Feasibility and validity of ecological momentary assessment in the investigation of suicide risk. Psychiatry Res 2014;220:564-70.

59 Whiting D, Fazel S. How accurate are suicide risk prediction models? asking the right questions for clinical practice. Evid Based Ment Heal 2019;22:125-8.

60 WHO. Suicide: one person dies every 40 seconds, 2019. Available: https://www.who.int/news-room/detail/09-09-2019-suicide-oneperson-dies-every-40-seconds [Accessed 9 Sep 2019].

61 Lopez-Castroman J, Blasco-Fontecilla H, Courtet P, et al. Are we studying the right populations to understand suicide? World Psychiatry 2015;14:368-9. 